\title{
A NOTE ON THE GENERALIZED JOSEPHUS PROBLEM
}

\author{
By \\ Saburô UCHIYAMA
}

1. This is a continuation, with supplementary notes, of our previous paper [3] in which some solutions have been provided for the generalized Josephus problem. If we are given two integers $m \geqq 2$ and $n \geqq 1$, supposing that $n$ objects, numbered from 1 to $n$, are arranged in a circle, starting then with object number 1 and counting each object in turn around the circle, clockwise or anticlockwise in the same direction fixed once for all, we eliminate every $m$ th object until all the objects are removed. The $k$ th Josephus number $a_{m}(k, n)(1 \leqq k \leqq n)$ is defined to be equal to $l(1 \leqq l \leqq n)$, if $l$ is the number attached to the object to be removed at the $k$ th step of reduction. We have plainly

$$
1 \leqq a_{m}(k, n) \leqq n
$$

and

$$
a_{m}(1, n) \equiv m \quad(\bmod n)
$$

Thus far there is no reason to exclude the value $m=1$, in which case we have trivially $a_{m}(k, n)=k(1 \leqq k \leqq n)$. For a given $m \geqq 1$ in general $m$ induces a permutation $\sigma_{m}=\sigma_{m, n}$ of the array $\langle 1,2, \ldots, n\rangle$, that is, the Josephus permutation

$$
\sigma_{m}=\left(\begin{array}{cccc}
1 & 2 & \cdots & n \\
a_{m}(1, n) & a_{m}(2, n) & \cdots & a_{m}(n, n)
\end{array}\right) .
$$

For a fixed integer $n \geqq 1$ the number of all possible, distinct permutations $\sigma_{m}$ for various values of $m$ equals $M_{n}$, the least common multiple (L.C.M.) of the integers $1,2, \ldots, n$, and this fact is an immediate consequence of the congruence substantially due to $P$. G. Tait,

$$
a_{m}(k+1, n+1) \equiv m+a_{m}(k, n) \quad(\bmod n+1)
$$

(cf. Proposition 1 below).

Received May 21, 2003.

Revised July 5, 2004. 
In $\S 2$ a description of the permutation $\sigma_{m}$ in terms of certain specific cyclic permutations will be given together with several interesting consequences thereof.

In the article [3] we have formulated a hypothesis on the infinitude of "limitative numbers" for every fixed $m$, suggested by Seki Takakazu (cf. [3]). A limitative number with respect to a given $m \geqq 2$ is by definition a positive integer $n$ satisfying the condition $d_{m}(n+1):=a_{m}(n+1, n+1)=1$. The hypothesis can be easily confirmed to be true for $m=2$ and 3 , as we have seen in [ $3 ; \S 7]$. For $m \geqq 4$ we may only prove that there are infinitely many positive integers $n$ satisfying the condition

$$
1 \leqq d_{m}(n+1) \leqq m-1 .
$$

A characterization of such integers $n$ will be given in $\S 3$ below.

It may be of some interest to note that a proposition dual to the hypothesis above, that is, the proposition to the effect that for every fixed integer $n \geqq 1$ there exist infinitely many positive integers $m$ such that $d_{m}(n+1)=1$, is easily shown to be true (Proposition 6).

Several Japanese mathematicians in the eighteenth century treated also a further generalization of the Josephus problem to determine Josephus numbers $a_{m}(k, n)$ in which the integer $m$ may be not necessarily the same in each step of eliminating the $n$ given objects. We shall present in $\S 4$ below an algorithm for determining the Josephus numbers $a_{(m)}(k, n)(1 \leqq k \leqq n)$ with an arbitrarily given sequence $(m)=\left(m_{1}, m_{2}, m_{3}, \ldots\right)$ of positive integers.

In this respect it will be convenient to call the integer $m$ in the Josephus problem, say, a reduction coefficient (脱数, as named by Seki in [2]). Thus we have so far considered the Josephus problem with a constant reduction coefficient, and may deal anew also with the problem with various sequences of reduction coefficients.

Thanks to the referee's useful comments the writer was able to improve the original draught of this paper.

2. Let us consider the generalized Josephus problem with given positive integers $m$ and $n$ as parameters. Here $m$ is at present a constant reduction coefficient and $n$ is the number of objects to be removed cyclically in turn by every $m$ th object. With the Josephus numbers $a_{m}(k, n)(1 \leqq k \leqq n)$ we set

$$
J_{m}=J_{m, n}=\left\langle a_{m}(1, n), a_{m}(2, n), \ldots, a_{m}(n, n)\right\rangle,
$$

which will be called the Josephus array corresponding to $m$. We may write symbolically 


$$
J_{m}=\sigma_{m} J_{1},
$$

where $J_{1}=\langle 1,2, \ldots, n\rangle$ and $\sigma_{m}$ is the Josephus permutation with respect to $m$ defined in $\S 1$.

If we set $M_{n}=$ L.C.M. $(1,2, \ldots, n)$ with $M_{1}=1$, then there holds

Proposition 1. There are exactly $M_{n}$ different Josephus permutations $\sigma_{m}=\sigma_{m, n}$ possible on $1,2, \ldots, n$.

There is nothing to prove for $n=1$. If $n=2$ then we have

$$
\sigma_{m}=\left(\begin{array}{ll}
1 & 2 \\
1 & 2
\end{array}\right) \text { or }\left(\begin{array}{ll}
1 & 2 \\
2 & 1
\end{array}\right)
$$

according as $m$ is odd or even. Supposing then that $n>2$ and writing

$$
\sigma_{m}=\left(\begin{array}{cccc}
1 & 2 & \cdots & n \\
l_{1} & l_{2} & \cdots & l_{n}
\end{array}\right) \text { and } \sigma_{m^{\prime}}=\left(\begin{array}{cccc}
1 & 2 & \cdots & n \\
l_{1}^{\prime} & l_{2}^{\prime} & \cdots & l_{n}^{\prime}
\end{array}\right),
$$

we see that if $m \equiv m^{\prime}\left(\bmod M_{n}\right)$ then by Tait's congruence (1) $l_{i}=l_{i}^{\prime}$ for $i=1,2, \ldots, n$, so that $\sigma_{m}=\sigma_{m^{\prime}}$. Suppose now that $m \neq m^{\prime}\left(\bmod M_{n}\right)$. If $m \not \equiv m^{\prime}(\bmod n)$ then $l_{1} \neq l_{1}^{\prime}$. If $m \equiv m^{\prime}(\bmod v)$ for $v=n, n-1, \ldots, n-v_{0}+1$ and $m \neq m^{\prime}\left(\bmod n-v_{0}\right)$ for some $v_{0}$ with $1 \leqq v_{0} \leqq n-2$, then $l_{i}=l_{i}^{\prime}$ for $i=$ $1,2, \ldots, v_{0}$ and $l_{v_{0}+1} \neq l_{v_{0}+1}^{\prime}$ again by the congruence (1), so that $\sigma_{m} \neq \sigma_{m^{\prime}}$.

REMARKS. 1) We have for $1 \leqq n \leqq 10$

\begin{tabular}{r|rrr}
$n$ & $n !$ & $M_{n}$ & $n ! / M_{n}$ \\
\hline 1 & 1 & 1 & 1 \\
2 & 2 & 2 & 1 \\
3 & 6 & 6 & 1 \\
4 & 24 & 12 & 2 \\
5 & 120 & 60 & 2 \\
6 & 720 & 60 & 12 \\
7 & 5040 & 420 & 12 \\
8 & 40320 & 840 & 48 \\
9 & 362880 & 2520 & 144 \\
10 & 3628800 & 2520 & 1440
\end{tabular}

Note that the relation

$$
\log M_{n}=(1+o(1)) n \quad(n \rightarrow \infty)
$$


is a consequence of (in reality, a relation equivalent to) the Prime Number Theorem.

The set $\mathbf{J}_{n}$ of all $M_{n}$ different Josephus permutations $\sigma_{m}=\sigma_{m, n}$ forms a group under ordinary multiplication of permutations for $1 \leqq n \leqq 4$, in fact, $\mathrm{J}_{n}=S_{n}(1 \leqq n \leqq 3)$ and $J_{4}=A_{4}$; for $n \geqq 5 \mathrm{~J}_{n}$ does not.

2) W. J. Robinson [1] has also considered Josephus permutations, by regarding the Josephus arrays which can be obtained from a given Josephus array by rotation as equivalent. However, this identification of the circular permutations is apparently inadequate for our present purposes. Our expressions to be furnished below for Josephus permutations (cf. Proposition 4) can nevertheless be compared with those of Robinson's.

We now define $M_{1,1}=1$ and

$$
M_{n, k}=\text { L.C.M. }(n, n-1, \ldots, n-k+1) \quad(1 \leqq k \leqq n) .
$$

Obviously we have $M_{n}=M_{n, n}$ and

$$
M_{n, 1}=n, \quad M_{n, 2}=n(n-1), \quad M_{n, k}=\text { L.C.M. }\left(n, M_{n-1, k-1}\right) .
$$

Proposition 2. For any given $n$ and $k(1 \leqq k \leqq n)$ we have

$$
a_{i}(k, n)=a_{j}(k, n) \quad \text { if } i \equiv j\left(\bmod M_{n, k}\right)
$$

and

$$
a_{i}(k, n)+a_{j}(k, n)=n+1 \quad \text { if } i \geqq 1, j \geqq 1 \text { and } i+j \equiv 1\left(\bmod M_{n, k}\right) .
$$

Proof. We have $a_{m}(1,1)=1$ trivially for all $m \geqq 1$. Suppose that $n \geqq 2$ and $1 \leqq k \leqq n$. The first assertion of the proposition is an immediate consequence of (1). As to the second we write

$$
j=z M_{n, k}+1-i \quad\left(1 \leqq i \leqq M_{n, k}\right),
$$

where $z$ denotes a positive integer not necessarily the same in each occurrence in the following. If we set

$$
a^{*}(k, n):=n+1-a_{j}(k, n),
$$

then with $j=z M_{n, 1}+1-i=z n+1-i$

$$
a^{*}(1, n)=n+1-a_{j}(1, n) \equiv i \quad(\bmod n),
$$

and with $j=z M_{n+1, k+1}+1-i$ 


$$
\begin{array}{rlr}
a^{*}(k+1, n+1) & =n+2-a_{j}(k+1, n+1) & \\
& \equiv n+2-\left(j+a_{j}(k, n)\right) & (\bmod n+1) \\
& \equiv i+\left(n+1-a_{j}(k, n)\right) & (\bmod n+1) \\
& \equiv i+a^{*}(k, n) & (\bmod n+1)
\end{array}
$$

since $M_{n+1, k+1} \equiv 0(\bmod n+1)$ and $\left(\bmod M_{n, k}\right)$. It follows that $a^{*}(k, n)=$ $a_{i}(k, n)$ in view of Tait's congruence (1). This completes the proof of our proposition.

Suppose now that $n \geqq 2$ and $1 \leqq k \leqq n$. Let $Z_{n, k}(l)$ denote the number of integers $m\left(1 \leqq m \leqq M_{n, k}\right)$ for which one has $a_{m}(k, n)=l(1 \leqq l \leqq n)$.

Proposition 3. For every $n \geqq 2$ and fixed $k(1 \leqq k \leqq n)$ we have

$$
Z_{n, k}(l)=\frac{M_{n, k}}{n}
$$

for each $l(1 \leqq l \leqq n)$.

Proof. If $k=1$ then we have for every $n$

$$
a_{m}(1, n) \equiv m \quad(\bmod n),
$$

whence follows

$$
Z_{n, 1}(l)=1=\frac{M_{n, 1}}{n}
$$

for any $l(1 \leqq l \leqq n)$.

Suppose then that $n$ and $k$ be given integers with $n \geqq k \geqq 2$ and $l$ $(1 \leqq l \leqq n)$ be as before any fixed integer and assume that we have $a_{m}(k, n)=l$ for some reduction coefficient $m\left(1 \leqq m \leqq M_{n, k}\right)$. Writing for simplicity's sake

$$
h_{i}=a_{m}(k-i, n-i) \quad(0 \leqq i \leqq k),
$$

where $h_{0}=l, 1 \leqq h_{i} \leqq n-i(1 \leqq i \leqq k-1)$ and $h_{k}=0$, we see in virtue of Tait's congruence (1) that the system of $k$ congruences

$$
m \equiv h_{i-1}-h_{i}(\bmod n-i+1) \quad(i=1,2, \ldots, k)
$$

admits a solution in $m\left(\bmod M_{n, k}\right)$. Since by our assumption any subsystem of the system $(2 ; k)$ is soluble in $m$, we may write $m=m_{n, j}\left(1 \leqq m_{n, j} \leqq M_{n, j}\right)$ for 
the solution of the system $(2 ; j)$ consisting of the first $j(1 \leqq j \leqq k)$ congruences in $(2 ; k)$. Note that the integer $m_{n, j}(1 \leqq j \leqq k)$ is uniquely determined (when the system $(2 ; j)$ is solvable) by the $j$-tuple of integers $\left(h_{1}, \ldots, h_{j}\right)$ and vice versa.

We are now going to evaluate the number $H_{j}(1 \leqq j \leqq k-1)$ of the possible choices of the values of $h_{j}$ when the other $h_{i}$ 's are chosen and fixed (with $h_{0}=l$ and $h_{k}=0$, of course).

We have for $j=1$

$$
m_{n, 1} \equiv h_{0}-h_{1} \quad\left(\bmod M_{n, 1}\right)
$$

and for $j=2$

$$
m_{n, 2} \equiv(1-n)\left(h_{0}-h_{1}\right)+n\left(h_{1}-h_{2}\right) \quad\left(\bmod M_{n, 2}\right),
$$

where the parameter $h_{1}\left(1 \leqq h_{1} \leqq n-1\right)$ may assume any integral value in the designated range, so that we have $H_{1}=n-1=(n-1) / d_{1}$ with $d_{1}=$ G.C.D. $\left(M_{n, 1}, n-1\right)=1$; here and in what follows we shall write

$$
d_{j}=\text { G.C.D. }\left(M_{n, j}, n-j\right) \text { for } 1 \leqq j \leqq k-1 .
$$

Suppose now that $k>j \geqq 2$. Then the system $(2 ; j+1)$ can be rewritten in the form

$$
(2 ; j+1) \quad \begin{cases}m \equiv m_{n, j} & \left(\bmod M_{n, j}\right) \\ m \equiv h_{j}-h_{j+1} & (\bmod n-j),\end{cases}
$$

where the solvability condition of this system is that

$$
m_{n, j} \equiv h_{j}-h_{j+1} \quad\left(\bmod d_{j}\right) .
$$

Let for $1 \leqq i \leqq k-1 L_{i}$ be an integer satisfying the condition $L_{i}\left(M_{n, i} / d_{i}\right) \equiv$ $1\left(\bmod (n-i) / d_{i}\right)$ and put $p_{i}=L_{i} M_{n, i} / d_{i}$. It is not difficult to verify in particular that

$$
m_{n, j} \equiv\left(1-p_{j-1}\right) m_{n, j-1}+p_{j-1}\left(h_{j-1}-h_{j}\right) \quad\left(\bmod M_{n, j}\right) .
$$

Since the integers $d_{j}$ and $d_{j-1}$ are coprime, $p_{j-1}$ is divisible by $d_{j}$, whence follows at once that $m_{n, j} \equiv m_{n, j-1}\left(\bmod d_{j}\right)$ and the solvability condition for the system $(2 ; j+1)$ is equivalent to

$$
m_{n, j-1} \equiv h_{j}-h_{j+1} \quad\left(\bmod d_{j}\right) ;
$$

thus the integer $h_{j}\left(1 \leqq h_{j} \leqq n-j\right)$ is uniquely determined to the modulus $d_{j}$ in terms of the other $h_{i}$ 's (in effect, of those $h_{i}$ 's for which $1 \leqq i \leqq j+1, i \neq j$ ) when they are appropriately specified. We have, therefore, $H_{j}=(n-j) / d_{j}$ whatever the 
actual values of the $h_{j}$ thus obtained may be, which naturally holds true for each $j(1 \leqq j \leqq k-1)$. We thus have proved that, if $n \geqq k \geqq 2$ and $Z_{n, k}(l)>0$, then there holds the relation

$$
Z_{n, k}(l) \leqq \prod_{j=1}^{k-1} H_{j}=\prod_{j=1}^{k-1} \frac{n-j}{d_{j}}=\frac{M_{n, k}}{n}
$$

which is trivially valid also if $Z_{n, k}(l)=0$; in here the inequality sign $\leqq$ must in fact be the equality $=$ for all $l(1 \leqq l \leqq n)$, since we have

$$
\sum_{l=1}^{n} Z_{n, k}(l)=M_{n, k}
$$

This completes the proof of our proposition.

Let $m, n$ and $k$ be again given integers such that $m \geqq 1, n \geqq 1$ and $1 \leqq k \leqq n$. We define $n$ cyclic permutations

$$
w_{r}=w_{r, n}:=(n-r+1, n-r+2, \ldots, n-1, n) \quad(1 \leqq r \leqq n) .
$$

Here $w_{1}=\sigma_{1}$ is always the identical permutation.

The next proposition gives a characterization of our Josephus permutations defined in $\S 1$; in there multiplication of two adjacent permutations should be performed from right to left, the associative law for the product of three or more permutations being naturally valid.

\section{Proposition 4. We have}

$$
\sigma_{m}=w_{n}^{m-1} w_{n-1}^{m-1} \cdots w_{2}^{m-1}
$$

where $\sigma_{m}$ is the Josephus permutation

$$
\sigma_{m}=\sigma_{m, n}=\left(\begin{array}{cccc}
1 & 2 & \cdots & n \\
a_{m}(1, n) & a_{m}(2, n) & \cdots & a_{m}(n, n)
\end{array}\right) .
$$

Proof is immediate, if we notice that

$$
w_{2}^{-m+1} \cdots w_{n-1}^{-m+1} w_{n}^{-m+1} \cdot \sigma_{1}=\left(\begin{array}{cccc}
a_{m}(1, n) & a_{m}(2, n) & \cdots & a_{m}(n, n) \\
1 & 2 & \cdots & n
\end{array}\right)=\sigma_{m}^{-1},
$$

which is nothing but the definition, or the actual formation, of the Josephus array $J_{m}=J_{m, n}$; the result (4) follows from this at once.

We note that Proposition 1 is an obvious consequence of Proposition 4. Also, it can be easily observed that the congruence relation (1) is a corollary of (4). In fact, we have by Proposition 4 


$$
J_{m, n}=w_{n}^{m-1}\left\langle 1, J_{m, n-1}+\langle 1\rangle_{n-1}\right\rangle
$$

where

$$
\left\langle 1, J_{m, n-1}+\langle 1\rangle_{n-1}\right\rangle=\left\langle 1, l_{1}+1, l_{2}+1, \ldots, l_{n-1}+1\right\rangle
$$

if $J_{m, n-1}=\left\langle l_{1}, l_{2}, \ldots, l_{n-1}\right\rangle$.

Notes. The cyclic permutation $w_{r}=(n-r+1, \ldots, n)(1 \leqq r \leqq n)$ is of length $r$. Suppose $r \geqq 2$ and determine the integer $s$ by the condition $m-1 \equiv s$ $(\bmod r), 0 \leqq s \leqq r-1$. If $s=0$ then

$$
w_{r}^{m-1}=w_{r}^{0}=w_{1}=\sigma_{1} \quad\left(\text { the identity of } S_{n}\right),
$$

and if $0<s \leqq r-1$ then, putting

$$
d_{r}:=\text { G.C.D. }(r, m-1) \text { and } t_{r}:=\frac{r}{d_{r}}
$$

we find

$$
w_{r}^{m-1}=w_{r}^{s}=\pi_{1} \pi_{2} \cdots \pi_{d_{r}}
$$

where

$$
\pi_{i}=\left(a_{i, 1}, a_{i, 2}, \ldots, a_{i, t_{r}}\right) \quad\left(1 \leqq i \leqq d_{r}\right)
$$

with $a_{i, j}\left(1 \leqq i \leqq d_{r}, 1 \leqq j \leqq t_{r}\right)$ determined by the conditions

$$
n-r+1 \leqq a_{i, j} \leqq n
$$

and

$$
a_{i, j} \equiv n+i+(j-1) s \quad(\bmod r)
$$

If $i \neq i^{\prime}$ then $\pi_{i}$ and $\pi_{i^{\prime}}$ have no common components.

By the way, the signature, or character, $\chi\left(\sigma_{m}\right)$ of the permutation $\sigma_{m}=\sigma_{m, n}$ is given by

$$
\chi\left(\sigma_{m}\right)=(-1)^{I} \quad \text { with } I=\frac{1}{2}(m-1) n(n-1) .
$$

A consequence of Proposition 4 is

Proposition 5. We have for $1 \leqq i \leqq M_{n}$

$$
\sigma_{M_{n}} \sigma_{i}=\sigma_{M_{n}+1-i}
$$


Here, it will suffice only to note that

$$
\sigma_{M_{n}}=\left(w_{2} \cdots w_{n-1} w_{n}\right)^{-1}=\left(\begin{array}{cccc}
1 & 2 & \cdots & n \\
n & n-1 & \cdots & 1
\end{array}\right) .
$$

We see from (5) that $d_{m}(n+1)=1$ whenever

$$
m \equiv 0 \quad\left(\bmod M_{n+1}\right) \text {. }
$$

Thus:

Proposition 6. Every integer $n \geqq 1$ is a limitative number with respect to infinitely many values of reduction coefficient $m$.

As a matter of fact, for every fixed $n \geqq 1$ the increasing sequence of positive integers $m$ satisfying the condition $d_{m}(n)=1$ possesses, by Proposition 3, asymptotic density $1 / n$.

For $n \geqq 2$ the cyclic permutations of $J_{1}=\langle 1,2, \ldots, n\rangle$ and of $J_{M_{n}}=\langle n$, $n-1, \ldots, 1>$ can be obtained in the following manner. If $d=$ G.C.D. $\left(n, M_{n-1}\right)$ then we have

$$
J_{1+z M_{n-1}}=w_{n}^{z M_{n-1}} J_{1} \quad\left(0 \leqq z \leqq \frac{n}{d}-1\right),
$$

which follows from Proposition 4, since

$$
\sigma_{1+z M_{n-1}}=w_{n}^{z M_{n-1}}
$$

and similarly

$$
J_{M_{n}-z M_{n-1}}=w_{n}^{z M_{n-1}} J_{M_{n}} \quad\left(0 \leqq z \leqq \frac{n}{d}-1\right)
$$

in view of Propositions 4 and 5.

3. Let $m$ be a fixed integer, $m \geqq 2$. We consider Josephus numbers $d_{m}(n):=$ $a_{m}(n, n)$ for various values of $n \geqq 1$, where $d_{m}(n)$ satisfies the relation (cf. (1))

$$
d_{m}(n+1) \equiv m+d_{m}(n) \quad(\bmod n+1)
$$

It follows from (6) that for $m \geqq 3$

$$
2 \leqq d_{m}(m) \leqq m-1 \quad \text { and } \quad d_{m}(m+1)=d_{m}(m)-1 \leqq m-2
$$


We now define a sequence of integers $n_{i}(i=1,2,3, \ldots)$ recursively by

$$
n_{1}=m \geqq 3, \quad n_{i+1}=\left\lfloor\frac{m\left(n_{i}+1\right)-d_{m}\left(n_{i}+1\right)}{m-1}\right\rfloor \quad(i \geqq 1) .
$$

Then it can be readily verified that

$$
1 \leqq d_{m}\left(n_{i}+1\right) \leqq m-1 \text { for all } i \geqq 1 .
$$

In fact, this sequence $n_{i}$ is nothing but the sequence $n_{i}$ defined in [3; §4] with $n_{1}=m$ and $c_{1}=d_{m}(m+1)$, and our analysis in there guarantees the validity of inequality (8); thus

Proposition 7. For every fixed reduction coefficient $m \geqq 3$ there are infinitely many positive integers $n$ satisfying the condition

$$
1 \leqq d_{m}(n+1) \leqq m-1
$$

the set of all such integers $n$ coincides with the set

$$
\{1,2, \ldots, m-1\} \cup\left\{n_{i}: i=1,2,3, \ldots\right\} .
$$

To ascertain the latter half of the statement of this proposition one may refer to our formula $(15)$ in $[3 ; \S 5]$.

For the sake of brevity we set $z_{i}=n_{i}+1(i \geqq 1)$. It follows from (7) that the $z_{i}$ satisfy the relation

$$
(m-1) z_{i+1}-m z_{i}=d_{m}\left(z_{i+1}\right)-d_{m}\left(z_{i}\right) \quad(i \geqq 1),
$$

and we find after a simple computation just as in $[3 ; \S 2]$

$$
\begin{aligned}
\theta & :=\lim _{j \rightarrow \infty} z_{j}\left(\frac{m-1}{m}\right)^{j}=z_{i}\left(\frac{m-1}{m}\right)^{i}+\sum_{v=i}^{\infty} \frac{d_{m}\left(z_{v+1}\right)-d_{m}\left(z_{v}\right)}{m}\left(\frac{m-1}{m}\right)^{v} \\
& =z_{i}\left(\frac{m-1}{m}\right)^{i}-\frac{d_{m}\left(z_{i}\right)}{m}\left(\frac{m-1}{m}\right)^{i}+\frac{1}{m-1} \sum_{v=i+1}^{\infty} \frac{d_{m}\left(z_{v}\right)}{m}\left(\frac{m-1}{m}\right)^{v}
\end{aligned}
$$

for all $i \geqq 1$, and this proves

PROPOSITION 8. We have for every fixed $m \geqq 3$ with $z_{i}=n_{i}+1$

$$
-\frac{m-2}{m}<z_{i}-\theta\left(\frac{m}{m-1}\right)^{i}<\frac{m-2}{m} \quad(i \geqq 1),
$$

where $\theta$ is the constant depending only on $m$ defined above. 
For $m=3$ and $4 z_{i}=n_{i}+1$ is thus determined to be the nearest integer to $\theta(m /(m-1))^{i}(i \geqq 1)$.
EXAMPLES. 1) $m=3: \quad \theta=2.73758147 \ldots$
2) $m=4: \quad \theta=3.88885089 \ldots$
3) $m=5: \quad \theta=5.00535701 \ldots$
4) $m=6: \quad \theta=5.78453860 \ldots$
5) $m=7: \quad \theta=6.79391843 \ldots$
6) $m=8: \quad \theta=7.98689276 \ldots$
7) $m=9: \quad \theta=8.66739501 \ldots$
8) $m=10: \quad \theta=9.64158446 \ldots$

4. As is noted in $\S 1$ above, it is known to us that some Japanese mathematicians in the Edo era treated the Josephus problem in such a still more generalized form as the reduction coefficient $m$ may vary in each step of eliminating the given objects. Thus we are given a number $n \geqq 2$ of the objects and a certain sequence of reduction coefficients $m_{v} \geqq 1(v=1,2,3, \ldots)$, and have to find for instance the number attached to the object which is to be removed at the specific $v$ th step, especially in the $n$th step, of reduction. The sequences $(m)=\left(m_{v}\right)$ of reduction coefficients we often observe in the existing literature (most of which has already become hardly accessible to us, however) are, for instance,

$$
\begin{aligned}
& \text { the natural numbers: } \quad m_{v}=v \\
& \text { the triangular numbers: } \quad m_{v}=\frac{1}{2} v(v+1) \text { and } \\
& \text { the tetrahedral numbers: } m_{v}=\frac{1}{6} v(v+1)(v+2) \text {. }
\end{aligned}
$$

Now, let $J_{1}=\langle 1,2, \ldots, n\rangle$ be as before the initial Josephus array. With the cyclic permutations $w_{r}=w_{r, n}(1 \leqq r \leqq n)$ specified in (3) we have

Proposition 9. The Josephus array $J_{(m)}$ of $n$ objects $1,2, \ldots, n$ with respect to a given sequence of reduction coefficients $(m)=\left(m_{v}\right)$ can be obtained through

$$
J_{(m)}=\sigma_{(m)} J_{1}
$$

where $\sigma_{(m)}$ is the permutation defined by

$$
\sigma_{(m)}=w_{n}^{m_{1}-1} w_{n-1}^{m_{2}-1} \cdots w_{2}^{m_{n-1}-1} .
$$


For $1 \leqq k<n$ (or rather $k<n-1$ ) the first $k$ components of $J_{(m)}$ are determined by the first $k$ factors (i.e. the $k$ factors counting from the left end) of this permutation $\sigma_{(m)}$.

Proof is similar to that of Proposition 4.

5. Here we collect some examples to our Propositions 4 and 9, illustrating the scope of the algorithms thereby implied.

Given an integer $n \geqq 2$ and a sequence of positive integers $(m)=\left(m_{v}\right)$, we set the starting array

$$
J^{(1)}=J_{1}=\langle 1,2, \ldots, n\rangle
$$

and successively determine the arrays

$$
J^{(s+1)}=w_{s+1}^{m_{n-s}-1} J^{(s)} \quad(1 \leqq s \leqq n-1)
$$

with the cyclic permutations $w_{r}$ defined in (3). The final array $J^{(n)}=J_{(m)}$ is the required Josephus array with respect to the sequence of reduction coefficients $\left(m_{v}\right)$.

In order only to determine the first $k$ components of the Josephus array $J_{(m)}$, where $1 \leqq k<n$, we may begin with $J^{(n-k)}=J_{1}$ and then deal with the arrays $J^{(s)}(n-k<s \leqq n)$ just as in the above.

Note that for $s(2 \leqq s<n)$ the first $n-s$ components of the array $J^{(s)}$ are regularly $1,2, \ldots, n-s$. In the examples that follow we shall, therefore, omit to enter these obvious numerals in the arrays relevant to our algorithms.

EXAMPLE 1. $n=16, m=10$

$$
\begin{aligned}
& J^{(2)}=〈 \\
& J^{(3)}=< \\
& J^{(4)}=< \\
& J^{(5)}=< \\
& J^{(6)}=< \\
& J^{(7)}=< \\
& J^{(8)}=< \\
& J^{(9)}=< \\
& J^{(10)}=< \\
& J^{(11)}=< \\
& J^{(12)}=< \\
& J^{(13)}=<
\end{aligned}
$$
$16,13,14,12,15>$ $14,13,16,11,15,12>$ $12,16,15,11,13,10,14\rangle$ $10,13,9,16,12,14,11,15\rangle$ $8,10,13,9,16,12,14,11,15\rangle$ $16,7,9,12,8,15,11,13,10,14\rangle$ $15,14,16,7,10,6,13,9,11,8,12\rangle$ $14,12,11,13,16,7,15,10,6,8,5,9\rangle$ $13,10,8,7,9,12,16,11,6,15,4,14,5\rangle$ 


$$
\begin{aligned}
& J^{(14)}=\langle\quad 12, \quad 8, \quad 5,3,16,4,7,11,6,15,10,13,9,14\rangle \\
& J^{(15)}=\langle\quad 11,6,2,14,12,10,13,16,5,15,9,4,7,3,8\rangle \\
& J^{(16)}=\langle 10,4,15,11,7, \quad 5,3,6,9,14,8,2,13,16,12,1\rangle=J_{10}
\end{aligned}
$$

EXAMPLE 2. $n=20, m_{v}=2 v-1(v \geqq 1)$

$$
\begin{aligned}
& J^{(2)}=〈 \\
& J^{(3)}=< \\
& J^{(4)}=< \\
& J^{(5)}=〈 \\
& J^{(6)}=< \\
& J^{(7)}=〈 \\
& J^{(8)}=< \\
& J^{(9)}=< \\
& J^{(10)}=< \\
& J^{(11)}=< \\
& J^{(12)}=< \\
& J^{(13)}=< \\
& J^{(14)}=< \\
& J^{(15)}=< \\
& J^{(16)}=< \\
& J^{(17)}=< \\
& J^{(18)}=< \\
& J^{(19)}=\langle 4,9,16,7,20,18,2,10,3,5,13,14,11,6,8,12,17,19,15\rangle \\
& J^{(20)}=\langle 1,4,9,16,7,20,18,2,10,3,5,13,14,11,6,8,12,17,19,15\rangle=J_{(m)} \\
& 17,19,20,18> \\
& 16,17,19,20,18\rangle \\
& 19,20,15,17,18,16\rangle \\
& 19,17,18,20,15,16,14\rangle \\
& 13,19,17,18,20,15,16,14\rangle \\
& 16,17,14,12,13,15,19,20,18\rangle \\
& 11,16,17,14,12,13,15,19,20,18> \\
& 17,18,12,13,10,19,20,11,15,16,14\rangle \\
& 13,9,10,16,17,14,11,12,15,19,20,18\rangle \\
& 9,14,10,11,17,18,15,12,13,16,20,8,19\rangle \\
& 19,7,12,8,9,15,16,13,10,11,14,18,20,17\rangle \\
& 16,14,17,7,18,19,10,11,8,20,6,9,13,15,12\rangle \\
& 13,8,6,9,15,10,11,18,19,16,12,14,17,5,7,20\rangle \\
& 10,19,14,12,15,4,16,17,7,8,5,18,20,6,11,13,9\rangle \\
& 7,14,5,18,16,19,8,20,3,11,12,9,4,6,10,15,17,13\rangle
\end{aligned}
$$

EXAMPLE 3. $n=20, m_{2 v-1}=9, m_{2 v}=13(v \geqq 1)$

$$
\begin{aligned}
& J^{(2)}=く \\
& J^{(3)}=く \\
& J^{(4)}=く \\
& J^{(5)}=く \\
& J^{(6)}=く \\
& J^{(7)}=く \\
& J^{(8)}=く \\
& J^{(9)}=く \\
& J^{(10)}=く \\
& J^{(11)}=く \\
& J^{(12)}=く
\end{aligned}
$$

$17,18,19,20\rangle$

$18,19,20,16,17\rangle$

$17,20,15,16,18,19\rangle$

$19,15,18,20,14,16,17\rangle$

$13,19,15,18,20,14,16,17\rangle$

$15,16,13,18,12,14,17,19,20\rangle$

$19,13,14,11,16,20,12,15,17,18>$

$11,20,14,15,12,17,10,13,16,18,19\rangle$

$17,19,16,10,11,20,13,18,9,12,14,15\rangle$ 


$$
\begin{aligned}
& J^{(13)}=\langle\quad 20,16,18,15,9,10,19,12,17,8,11,13,14\rangle \\
& J^{(14)}=< \\
& 15,14,10,12,9,17,18,13,20,11,16,19,7,8\rangle \\
& J^{(15)}=< \\
& J^{(16)}=< \\
& J^{(17)}=< \\
& J^{(18)}=< \\
& J^{(20)}=\langle 9 \text {, } \\
& 18,12,11,7,9,6,14,15,10,17,8,13,16,19,20\rangle \\
& 13,10,20,19,15,17,14,6,7,18,9,16,5,8,11,12\rangle \\
& 16,8,5,15,14,10,12,9,18,19,13,4,11,17,20,6,7\rangle \\
& 11,6,16,13,5,4,18,20,17,8,9,3,12,19,7,10,14,15\rangle \\
& 14,4,18,9,6,17,16,11,13,10,20,2,15,5,12,19,3,7,8\rangle
\end{aligned}
$$

EXAMPLE 4. $n=20, m_{v}=\frac{1}{2} v(v+1)(v \geqq 1)$

$$
\begin{aligned}
& J^{(2)}=< \\
& J^{(3)}=< \\
& J^{(4)}=< \\
& J^{(5)}=< \\
& J^{(6)}=< \\
& J^{(7)}=< \\
& J^{(8)}=< \\
& J^{(9)}=< \\
& J^{(10)}=< \\
& J^{(11)}=< \\
& J^{(12)}=< \\
& J^{(13)}=< \\
& J^{(14)}=< \\
& J^{(15)}=< \\
& J^{(16)}=< \\
& J^{(17)}=< \\
& J^{(18)}=< \\
& J^{(19)}=\langle 4,10,20,18,7,6,19,14,13,5,16,3,2,17,8,9,15,12,11\rangle \\
& J^{(20)}=\langle 1,4,10,20,18,7,6,19,14,13,5,16,3,2,17,8,9,15,12,11\rangle=J_{(m)} \\
& 20,19,18> \\
& 17,20,19,18\rangle \\
& 16,17,20,19,18> \\
& 20,15,16,19,18,17\rangle \\
& 20,19,14,15,18,17,16> \\
& 15,14,13,16,17,20,19,18> \\
& 17,20,19,18,12,13,16,15,14\rangle \\
& 16,12,15,14,13,17,18,11,20,19> \\
& 20,15,11,14,13,12,16,17,10,19,18> \\
& 17,16,11,19,10,9,20,12,13,18,15,14\rangle \\
& 17,13,12,20,15,19,18,16,8,9,14,11,10\rangle \\
& 20,16,12,11,19,14,18,17,15,7,8,13,10,9\rangle \\
& 11,10,6,17,16,9,19,8,7,20,12,13,18,15,14\rangle \\
& 19,9,8,20,15,14,7,17,6,5,18,10,11,16,13,12\rangle \\
& 13,11,18,17,12,7,6,16,9,15,14,10,19,20, .8,5,4\rangle \\
& 8,18,16,5,4,17,12,11,3,14,20,19,15,6,7,13,10,9\rangle
\end{aligned}
$$

EXAMPLE 5. $n=16, m_{v}=\frac{1}{6} v(v+1)(v+2)(v \geqq 1)$

$$
\begin{aligned}
& J^{(2)}=く \\
& J^{(3)}=く \\
& J^{(4)}=く \\
& J^{(5)}=く \\
& J^{(6)}=く \\
& J^{(7)}=く
\end{aligned}
$$

$16,15\rangle$

$15,14,16>$

$15,13,16,14>$

$15,13,16,14,12\rangle$

$14,12,16,13,11,15\rangle$

$12,16,14,11,15,13,10\rangle$ 


$$
\begin{aligned}
& J^{(8)}=< \\
& J^{(9)}=< \\
& J^{(10)}=< \\
& J^{(11)}=< \\
& J^{(12)}=< \\
& J^{(13)}=< \\
& J^{(14)}=< \\
& J^{(15)}=< \\
& J^{(16)}=\langle 1,5,15 \\
& 12, \quad 5, \quad 3,4,9,13,7,11,6,15,10,16,14,8\rangle \\
& 15,16,8,11,6,10,5,13,9,14,12,7\rangle \\
& 10,13,8,12,7,15,11,16,14,9\rangle \\
& 6,10,13,8,12,7,15,11,16,14,9\rangle \\
& 10,8,9,14,4,12,16,11,6,15,7,5,13\rangle \\
& 15,8,6,7,12,16,10,14,9,3,13,4,2,11\rangle \\
& 5,8, \quad 6,7,12,16,10,14,9,3,13,4,2,11\rangle=J_{(m)}
\end{aligned}
$$

\section{References}

[1] Robinson, W. J., The Josephus problem, Math. Gazette 44 (1960), 47-52.

[2] Seki Takakazu, 算脱之法 (1683). Takakazu Sekı's Collected Works, edited with expls. by Akira Hirayama et al., Osaka, 1974: pp. 211-214.

[ 3 ] Uchiyama, S., On the generalized Josephus problem, Tsukuba J. Math. 27 (2003), 319-339.

Saburô Uchiyama

Prof. Emer.

University of Tsukuba

(Institute of Mathematics)

and

Okayama University 